\title{
Neurology Research in Saudi Arabia
} Urgent call for action

"Hussein Algahtani, ${ }^{1,2}$ Bader Shirah, ${ }^{3}$ Faisal Boker, ${ }^{1}$ Albaraa Algamdi, ${ }^{1}$ Abdulah Alkahtani ${ }^{4}$

$$
\text { أبحاث طب الأعصاب في المملكة العربية السعودية }
$$

حسين القحطاني، بدر شيره، فيصل بوكر، البراء الغامدي، عبداله القحطاني

ABSTRACT: Objectives: Research activities in Saudi Arabia are promoted at the governmental and institutional levels. However, the output and quality of research conducted in the field of neurology has not yet been measured quantitatively. This study therefore aimed to analyse neurology-related publications from Saudi Arabia. Methods: This study was conducted in January 2016. A systematic search using the PubMed ${ }^{\circledR}$ search engine (National Library of Medicine, Bethesda, Maryland, USA) was conducted to identify all neurology-related articles published from Saudi Arabia between January 1996 and December 2015. Results: A total of 1,292 neurologyrelated publications were identified. Neurology research increased linearly with time, with most publications originating from Riyadh $(67 \%)$ and the university sector $(\approx 47 \%)$. However, most neurology-related articles were published in journals which had an impact factor of $<1$ (55\%). Conclusion: Neurology research in Saudi Arabia has increased substantially over the last 20 years. However, as most articles were published in low-impact journals, the quality of research remains inadequate and should be improved. It is important that an official research culture be established in both governmental and private universities as well as colleges and health institutions in Saudi Arabia. The formation of clinical academic departments staffed by research experts is recommended to ensure the quality of neurology research output.

Keywords: Neurology; Research; Publications; Journal Impact Factor; Saudi Arabia.

الملخص: الهدف: إن الأنشطة البحثية في المملكة العربية السعودية عادة ما تكون مدعومة الأنية من قبل المؤسسات الحكومية بشتى أنواعها.

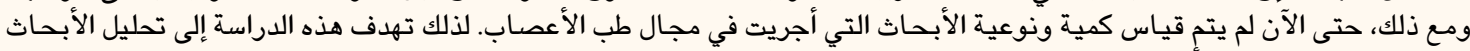

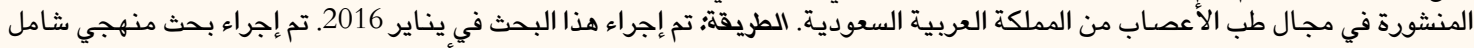

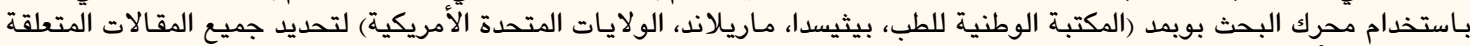

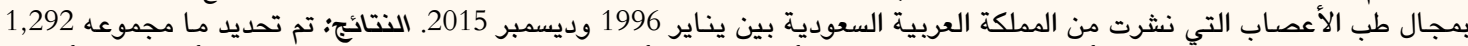

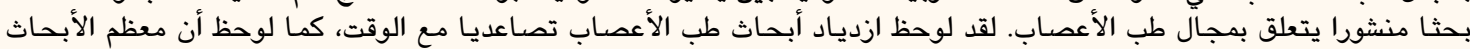

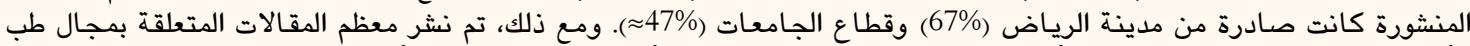

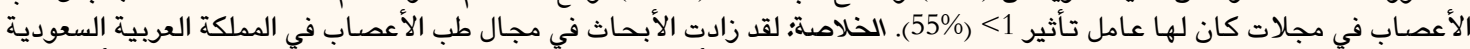

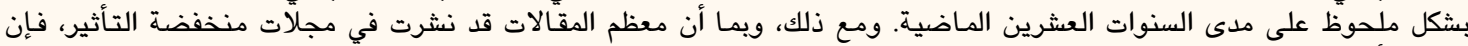

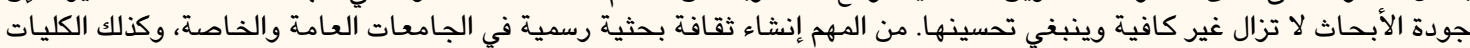

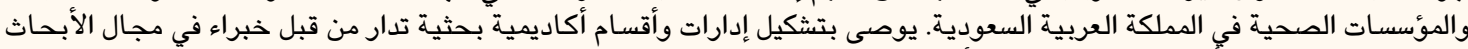

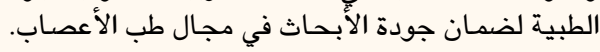

الكلمات المفتاحية: طب الأعصاب؛ بحث؛ المنشورات؛ عامل تأثير المجلة؛ المملكة العربية السعودية.

\section{AdvanCES IN KNOWLEDGE}

This study analyses neurology-related articles published from Saudi Arabia between January 1996 and December 2015, highlighting the status of past and current neurology research in the country.

The findings of this study indicate that research culture urgently needs to be promoted in Saudi Arabia, perhaps by establishing clinical academic departments staffed by research experts.

\section{Application to Patient Care}

The results of this study may encourage institutions in Saudi Arabia to promote research and research methodology education. The subsequent increase in high-quality neurology-related publications would support medical practice and patient care by improving understanding of disease trends and risk factors as well as informing management guidelines for diseases and conditions specific to Saudi Arabia. 
$\mathrm{P}$ UBLISHING A PAPER IS THE MOST COMMON way of communicating the results of research to other researchers, health professionals and members of the scientific community, with publications in international peer-reviewed journals usually seen as an indicator of proper scientific research. ${ }^{1}$ In Saudi Arabia, research activities are promoted at the governmental and institutional levels. ${ }^{2}$ Nevertheless, clinical neuroscience research productivity in Saudi Arabia still lags behind that of other countries. ${ }^{3}$ To the best of the authors' knowledge, the quantity and quality of research conducted in the field of neurology in Saudi Arabia has not yet been measured quantitatively. Therefore, this study was conducted to analyse neurology-related publications from Saudi Arabia between January 1996 and December 2015.

\section{Methods}

This study was conducted in January 2016. A search of all neuroscience-related articles from Saudi Arabia published between January 1996 and December 2015 was performed using the PubMed ${ }^{\circledR}$ search engine (National Library of Medicine, Bethesda, Maryland, USA). This search engine was selected due to its specialisation in medical literature, the feasibility of use of its Boolean operator search function and MEdical Subject Headings (MeSH) classifications and the increased likelihood of compiling the largest number of eligible publications due to the huge number of records available from publisher-supplied citations and the MEDLINE ${ }^{\circledR}$ (National Library of Medicine) database.

The search strategy involved 206 terms deemed relevant and specific to the field of neuroscience which were combined using the Boolean operators "and" and "or", with Saudi Arabia designated as the country of affiliation. The National Library of Medicine MeSH terms were used to ensure that the search was comprehensive. The inclusion criteria consisted of publications conducted in Saudi Arabia with the primary author affiliated with a Saudi Arabian institution and the study sample or population consisting of Saudi nationals or residents. In addition, only original articles, reviews, case reports or case series were included while abstracts of meetings, news items, book reviews, communications, letters, editorials and supplementary articles were excluded. Duplicate articles and false-positive neuroscience publications were also excluded.

Overall, the literature search revealed 5,626 neuroscience-related publications affiliated with Saudi Arabia. Subsequently, two consultant neurologists independently screened the titles and abstracts of these publications for suitability. In total, 3,251 falsepositive articles and 142 duplicate publications were excluded. Another 210 and 156 publications were excluded due to article type and because they did not meet the inclusion criteria, respectively. The 2,009 remaining publications were categorised based on their subspecialty, which revealed 1,292 articles in neurology, 299 in neurosurgery, 155 in psychiatry and 263 in basic sciences subspecialties [Figure 1]. For all neurology-related articles, information was collected related to study design, institution affiliations, authors, year of publication, city of origin, journal and journal impact factor (IF).

\section{Results}

A total of 1,292 neurology-related articles from Saudi Arabia were identified. Neurology research production showed a clearly increasing linear trend over time, with the greatest number of articles published in 2014

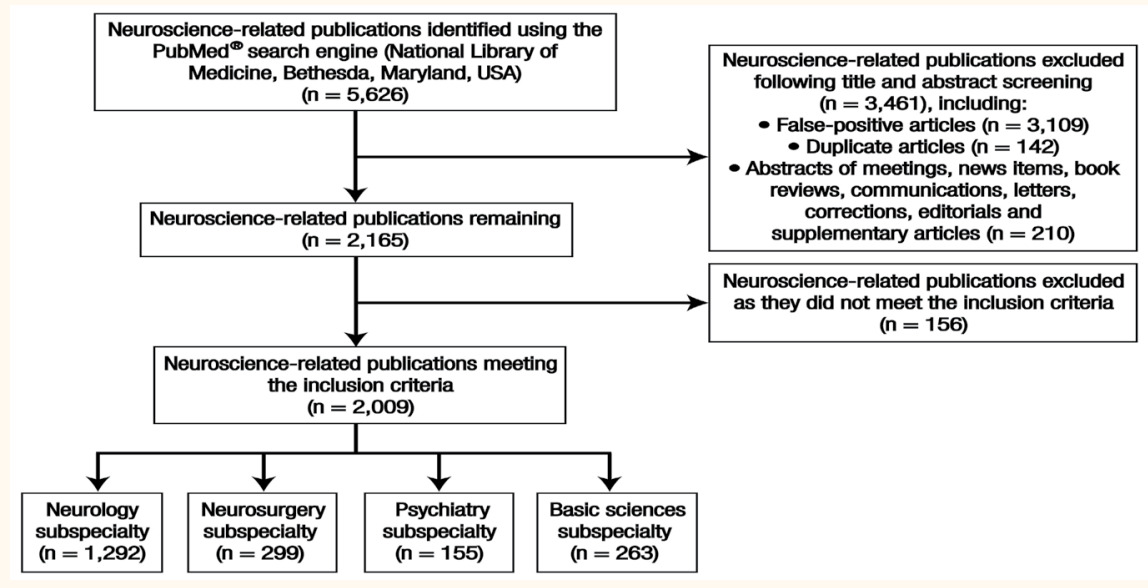

Figure 1: Diagram illustrating the search strategy used to identify neurology-related articles published from Saudi Arabia between January 1996 and December 2015. 


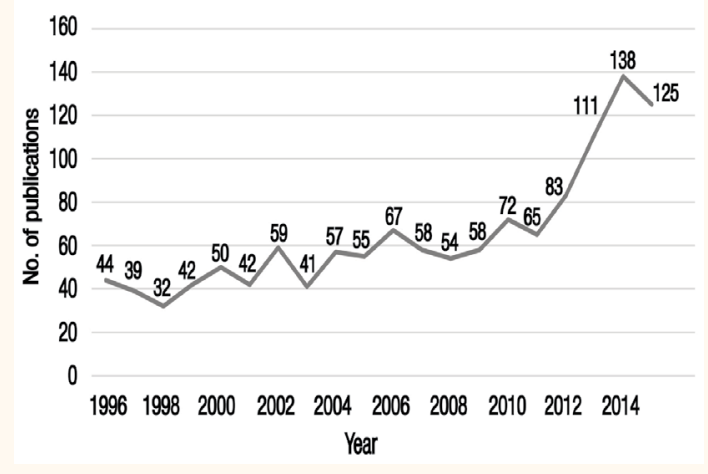

Figure 2: Number of neurology-related publications from Saudi Arabia between January 1996 and December 2015 per year $(\mathrm{N}=1,292)$.

and 2015 (138 and 125 articles, respectively) [Figure 2] . Most neurology publications were from Riyadh (67\%), the capital of Saudi Arabia, followed by Jeddah (15\%). Other cities with contributions to neurology research output included Al-Khobar (4\%), Dammam (3\%), Abha (2\%), Medina (1\%), Mecca (1\%), Dhahran (1\%) and Hofuf (1\%). The remaining cities had few or no neurology-related publications [Figure 3].

Approximately $47 \%$ of all neurology-related publications were affiliated with the university sector. The King Faisal Specialist Hospital and Research Centre was the most prolific institution, accounting for $23 \%$ of all publications. Among universities, the King Saud University and King Abdulaziz University published $21 \%$ and $9 \%$ of publications, respectively [Figure 4]. Overall, 55\% of articles were published in journals with an IF of $<1$ and $1 \%$ were published in journals without an IF. Only $2 \%$ of papers were published in journals with an IF of $\geq 7$ [Figure 5]. Among all of the articles analysed, the publishing journal with the highest IF was the Lancet, which had an IF of 44.002 in $2015 .^{4}$

In terms of research design, randomised controlled trials (RCTs) constituted only $4 \%$ of all publications;

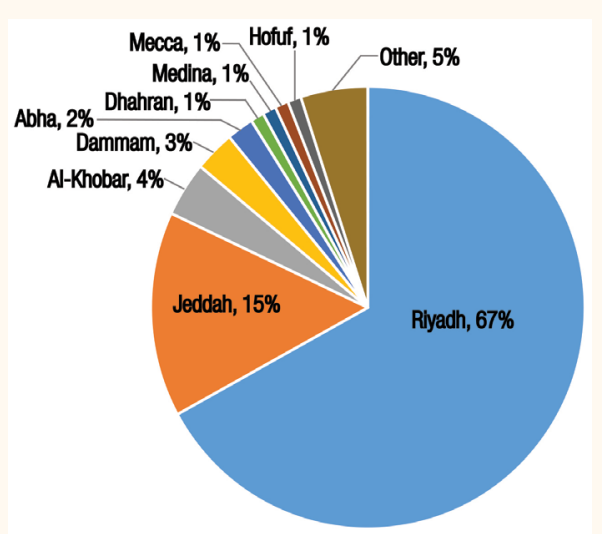

Figure 3: Percentage of neurology-related publications from Saudi Arabia between January 1996 and December 2015 by city $(\mathrm{N}=1,292)$.

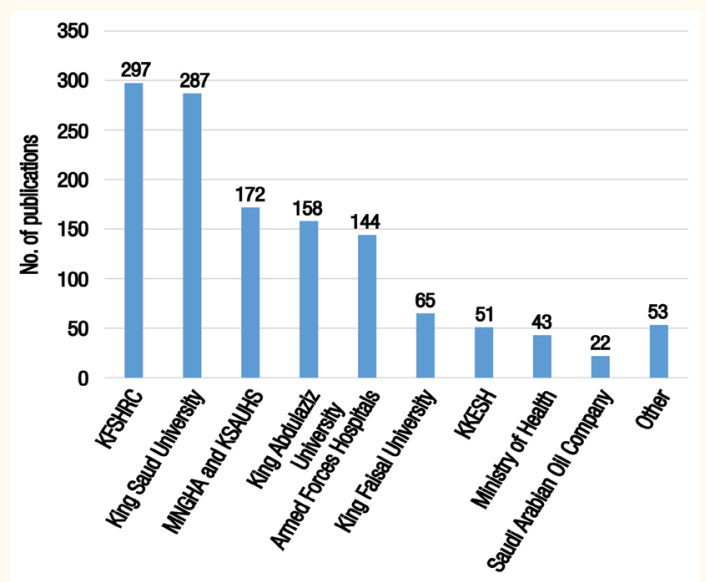

Figure 4: Number of neurology-related publications from Saudi Arabia between January 1996 and December 2015 by institution $(\mathrm{N}=1,292)$.

KFSHRC = King Faisal Specialist Hospital and Research Center; MNGHA = Ministry of National Guard Health Affairs; KSAUHS = King Saud bin Abdulaziz University for Health Sciences; KKESH = King Khaled Eye Specialist Hospital.

the remaining articles were case reports (40\%), retrospective cohort studies (18\%), reviews (15\%), prospective cohort studies (14\%), cross-sectional studies (5\%) or case series (4\%) [Figure 6]. The most common neurology research subspecialties were infectious diseases (15\%), paediatrics (13\%), epilepsy (12\%), genetics (10\%) and stroke research (10\%). Surprisingly, very few articles were published on cognitive and behavioural neurology and neuroimmunology (i.e. multiple sclerosis and neurorehabilitation).

\section{Discussion}

This study highlights the status of neurology research and types of neurology-related articles published in Saudi Arabia between January 1996 and December 2015. These findings indicate where Saudi Arabia stands in terms of past and present

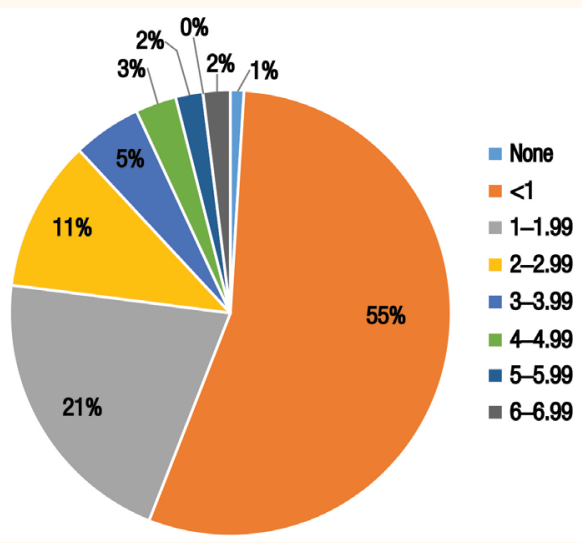

Figure 5: Percentage of neurology-related publications from Saudi Arabia between January 1996 and December 2015 by journal impact factor $(\mathrm{N}=1,292)$. 


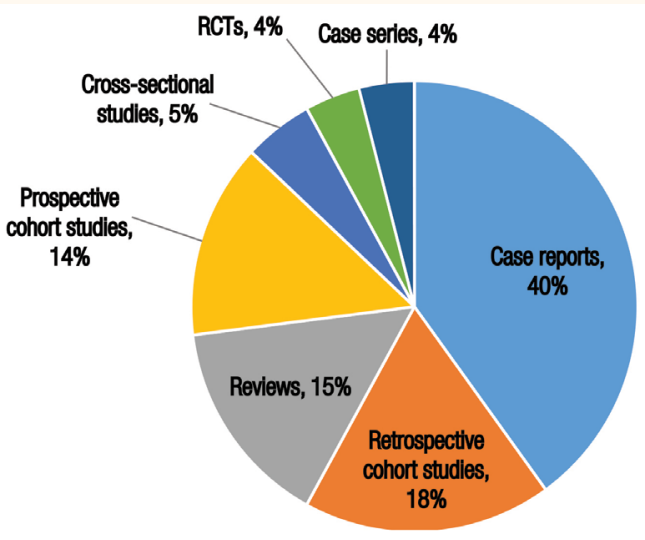

Figure 6: Percentage of neurology-related publications from Saudi Arabia between January 1996 and December 2015 by research design $(\mathrm{N}=1,292)$.

$R C T s=$ randomised controlled trials .

research production and should be considered an urgent call for action. Neurology research showed a clear linear increase over the past two decades; this may be attributable to realisation on the part of the Saudi Arabian government and its universities and medical institutes of the importance of such research in improving quality-of-life and long-term sustainable development. In addition, the growth in the number of published scientific papers in the field of neurology may also be due to the recent increase in the number of tertiary care centres and universities, the return of highly educated Saudi neurologists from abroad and the establishment of scientific journals based in Saudi Arabia. According to the Ministry of Education, Saudi Arabia is currently recognised as a regional leader in science, including medicine; in addition, it is forecast to become an Asian power by 2020 and a knowledgebased advanced industrial nation by $2025 .{ }^{5}$

However, the research designs of most neurologyrelated publications identified in the current study were found to be of low quality and importance, with an absence of well-designed RCTs and most articles consisting of case reports, retrospective cohort studies, reviews and prospective cohort studies. Moreover, more than half of the identified articles were published in journals with an IF of $<1$ and only $2.3 \%$ were published in journals with an IF of $\geq 7$. These findings suggest that there is a lack of support for research in Saudi Arabia and that further action is required to promote the publication of Saudi Arabian research in high-impact medical journals. As such, one recommendation would be to establish clinical academic departments in institutions so as to ensure and monitor the quality of research output.

In the current study, most neurology-related publications originated from the two largest cities in Saudi Arabia-Riyadh and Jeddah-with very little contribution from other cities. Tadmouri et al. similarly observed that the vast majority of biomedical research from Saudi Arabia between 1982-2000 originated from Riyadh, with all other cities together accounting for only $30 \%$ of the total research output. ${ }^{6}$ Despite the recent establishment of new universities, colleges, hospitals and research centres in other Saudi Arabian cities, their contribution to research remains minimal. Moreover, the relative contribution of different sectors indicated that most neurology research was conducted by universities, with the Saudi Arabian Ministry of Health and private hospitals producing very few papers. In addition, newer universities were found to produce fewer neurology-related publications, with most originating from the more established King Saud University and King Abdulaziz University. It could be argued that the newer institutions were created principally to educate medical students and provide health services to the local population rather than to conduct research; however, the authors of the current study believe that all medical colleges should actively engage in research and that medical education should be provided hand-in-hand with research training. Furthermore, the promotion of academic staff at universities often depends upon the publication of a certain number of articles in scientific journals. Close monitoring and strict regulations for academic promotions at Saudi Arabian institutions should therefore be implemented to encourage the publication of high-quality research.

To the best of the authors' knowledge, this study is the first to analyse neurology research productivity in Saudi Arabia. In order to avoid inaccuracies, each article was screened manually to exclude false-positive results and articles with duplicated content in multiple journals. However, the quantitative analysis was limited to neurology-related publications only and primarily addressed frequency of publication; the quality of the research or its benefits to the medical field or general public were not analysed. Further studies are therefore recommended to analyse these aspects of neurology research productivity in Saudi Arabia.

\section{Conclusion}

While neurology research in Saudi Arabia was found to have increased over the last 20 years, most articles were published in journals with low IFs. Thus, the quality of research publications must be improved. It was also noted that most research was conducted by government-run institutions, with few contributions from private institutions. It is therefore vital that the importance of establishing a research culture in governmental and private universities, colleges and 
health institutions be promoted so as to increase research productivity in the country. To this end, the authors recommend the establishment of clinical academic departments at higher education institutions to promote and monitor research output.

\section{CONFLICT OF INTEREST}

The authors declare no conflicts of interest.

\section{FUNDING}

No funding was received for this study.

\section{References}

1. Macilwain C. Science economics: What science is really worth. Nature 2010; 465:682-4. doi: 10.1038/465682a.

2. Meo SA, Hassan A, Usmani AM. Research progress and prospects of Saudi Arabia in global medical sciences. Eur Rev Med Pharmacol Sci 2013; 17:3265-71.

3. Jamjoom AB. State of clinical neuroscience research in Saudi Arabia: Where do we rank in the world? Neurosciences (Riyadh) 2016; 21:268-70. doi: 10.17712/nsj.2016.3.20160001.

4. Science Citation Index. Lancet impact factor. From: www.scijournal. org/impact-factor-of-LANCET.shtml Accessed: Mar 2017.

5. Latif R. Medical and biomedical research productivity from the Kingdom of Saudi Arabia (2008-2012). J Family Community Med 2015; 22:25-30. doi: 10.4103/2230-8229.149583.

6. Tadmouri GO, Tadmouri NB. Biomedical research in the Kingdom of Saudi Arabia (1982-2000). Saudi Med J 2002; 23:20-4 